\title{
Post-cholecystectomy acute injury: What can go wrong?
}

\author{
Vikas Gupta ${ }^{1}$, Ashish Gupta ${ }^{1}$, Thakur Deen Yadav $^{1}$, Bhagwant Rai Mittal ${ }^{2}$, and Rakesh Kochhar ${ }^{3}$ \\ Departments of ${ }^{1}$ General Surgery, ${ }^{2}$ Nuclear Medicine and ${ }^{3}$ Gastroenterology, \\ Postgraduate Institute of Medical Education and Research, Chandigarh, India
}

\begin{abstract}
Backgrounds/Aims: Most of the emphasis of postcholecystectomy injuries is laid on iatrogenic bilary trauma. However, they can involve a wide spectrum of injuries. Methods: We prospectively evaluated 42 patients with postcholecystectomy injuries referred to us from July 2011 to December 2012. Based on spectrum of injuries, we proposed an algorithm of management. Results: Injuries occurred following laparoscopy in 20 (2 converted) patients and open in 22 patients. Mean time of detection of injury was $4.32 \pm 2.33$ days. The nature of drainage was bilious in 36 , bile with blood in 2 , only blood in 2, and enteric in 2. Nine had organ failure at presentation. Six (14\%) needed re-operation. Source of hemorrhage was from right hepatic artery in three and small bowel mesentry in 1. Enteric injuries were one each to duodenum and colon. Six patient $(14 \%)$ died. Advancing age and organ failure were the predictors of mortality. Persistant biliary fistula was seen in $5(14 \%)$. Ten had lateral leaks that closed at $28.89 \pm 2.34$ days. Twenty-two formed stricture which was successfully managed with definitive hepaticojejunostomy. Conclusions: Post cholecystectomy acute injury does not limit itself to bile duct or vascular injury but it can traumatize adjacent hollow viscus or mesentery. It is important to diagnose and intervene enteric injury early. Presentation and management for such injury should be followed as per the proposed algorithm. (Ann Hepatobiliary Pancreat Surg 2019;23:138-144)
\end{abstract}

Key Words: Acute; Bile duct; Injury; Cholecystectomy; Vascular; Enteric; Stricture; Fistula

\section{INTRODUCTION}

Laparoscopic cholecystectomy was introduced three decades ago. Since then, it has been established as a gold standard treatment for symptomatic calculous cholecystitis. However, it is associated with three times the higher incidence of bile duct injuries when compared to open procedures. $^{1,2}$ Although the reported incidence remains less than $1 \%$, still it causes a substantial burden to the health care system due to large number of cholecystectomies performed across the globe. ${ }^{1-4}$

Consequences of bile duct injury can be severe. Besides early complications, which can be life threatening, there is always a chance of delayed stricture formation. Despite having a good repair of biliary stricture, the patients have to be kept on long-term follow-up for the development of late complications. ${ }^{5,6}$ In addition, these injuries lead on to economic burden to the society and are also responsible for high rates of medico-legal claims. ${ }^{7,8}$
There are many reports regarding the outcomes and management of biliary strictures ${ }^{5,6,9}$ but there is a paucity of literature pertaining to the outcome of injuries in acute setting. The present report focuses on the spectrum and outcome of post cholecystectomy acute injuries.

\section{MATERIALS AND METHODS}

From July 2011 to December 2012, 42 consecutive patients with acute biliary injury following cholecystectomy were prospectively studied at Postgraduate Institute of Medical Education and Research, Chandigarh, a tertiary care center in north India. The study was approved by the Institute ethics committee (IRB number: 8723/PGI/2Trg/ 11/15057). Informed consent was taken from all patients prior to enrolment. Patients without biliary fistula who underwent early repair were excluded from this study. Attempt was made to find details of cholecystectomy as recorded in the written referral note or by telephonic con-

Received: November 22, 2018; Revised: February 18, 2019; Accepted: February 21, 2019

Corresponding author: Vikas Gupta

Department of General Surgery, Postgraduate Institute of Medical Education and Research, Chandigarh 160012, India Tel: +91-172-2756645, Fax: +91-172-2744401, E-mail: vikaspgi@gmail.com

Copyright (C) 2019 by The Korean Association of Hepato-Biliary-Pancreatic Surgery

This is an Open Access article distributed under the terms of the Creative Commons Attribution Non-Commercial License (http://creativecommons.org/ licenses/by-nc/4.0) which permits unrestricted non-commercial use, distribution, and reproduction in any medium, provided the original work is properly cited. Annals of Hepato-Biliary-Pancreatic Surgery • pISSN: 2508-5778 - elSSN: 2508-5859 
versation with the operating surgeon. Patients were evaluated with detailed clinical examination, with relevant biochemical, hematological and coagulation parameters.

Post cholecystectomy biliary fistula was defined as an abnormal leak of bile from biliary system occurring within 30 days of cholecystectomy. Controlled fistula was defined as efflux of bile outside the abdominal cavity through a drain, without getting collected inside without any evidence of sepsis. Persistent fistula was defined as a fistula persisting beyond 3 months after cholecystectomy without showing any decreasing trend. Closure of fistula was defined as when drain output was minimal and nonbilious without any identifiable intra-abdominal collection on imaging in the absence of sepsis.

\section{Management protocol}

Patients were resuscitated with crystalloid and colloids as per the requirement. Empiric broad spectrum antibiotics were started. Abdominal ultrasonogram and Computed tomogram was done to establish collections. Note was made of the content of the abdominal drain (if placed previously) and management strategy was established accordingly (Fig. 1, flow chart). Intra-abdominal collections were drained with image guidance or by re-operation as per the clinical need, so as to establish controlled fistula. Once the fistula was controlled HIDA scan was done to establish the bilio enteric continuity. Patients with bilio-enteric continuity were subjected to endoscopic therapy.

Patients were followed up and time taken for closure of fistula was noted. All patients were evaluated for the development of stricture and for definitive management. Injuries were classified as per Strasberg classification. ${ }^{10}$ Definitive surgical repair was contemplated in patients with biliary stricture. All the patients with persistence of fistula beyond 3 months underwent definitive surgical repair as required. The patients were followed up with periodic clinical assessment and liver function test (LFT). Repeat imaging was carried out in case of recurrent cholangitis or deranged LFT. The last follow up was obtained

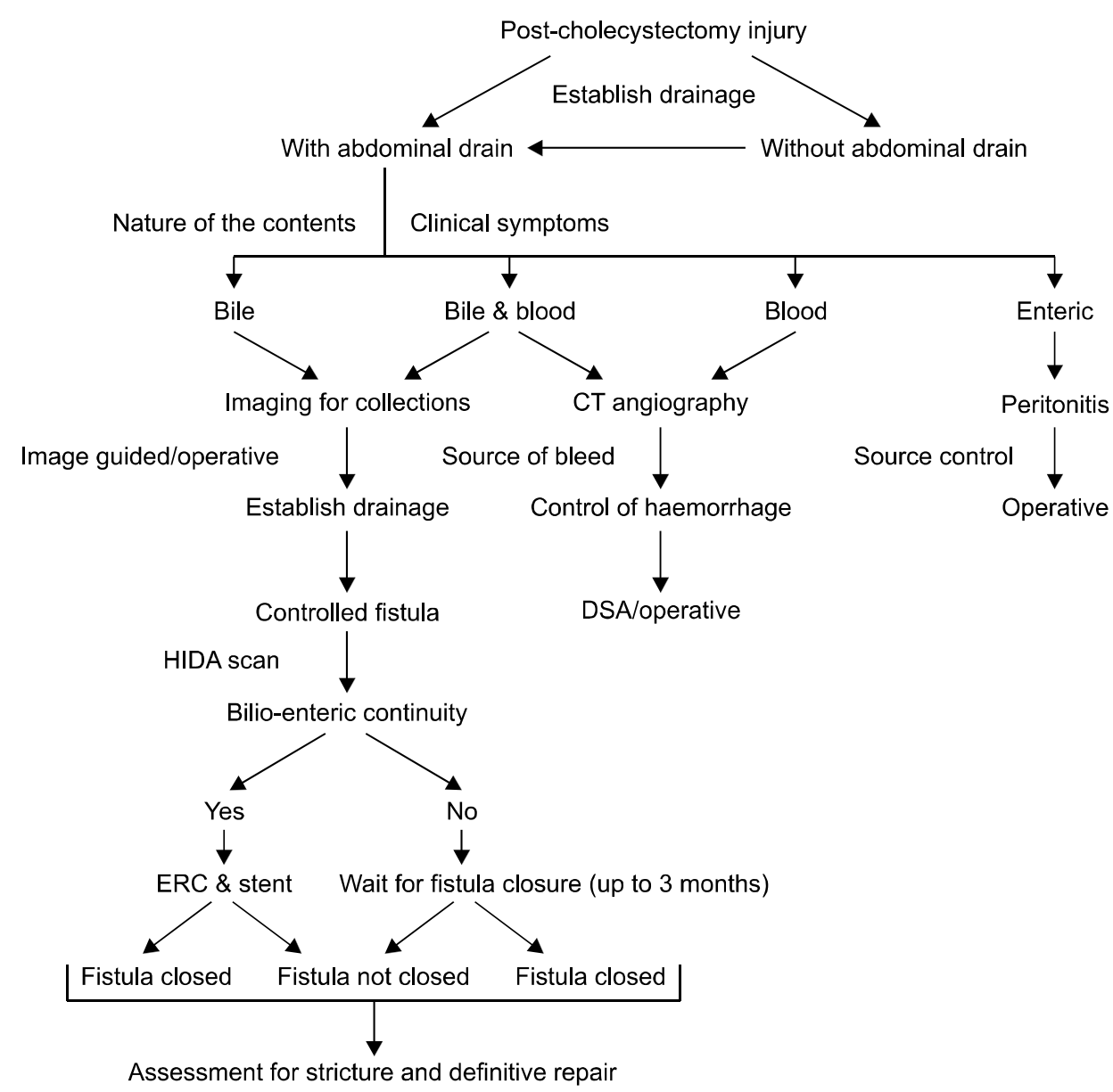

Fig. 1. Flow chart to show the management protocol. 
till December 2017.

\section{RESULTS}

The mean age of 42 patients was $41.88 \pm 14.56$ (range: 13-86) years. The male to female ratio was $1: 4.25$. Fortyone underwent cholecystectomy at primary or secondary health care level prior to referral while one had cholecystectomy done at our institute. During this period a total of 162 cholecystectomies were performed, and we report an incidence of $0.61 \%$ of bile duct injury.

\section{Scenario of cholecystectomy and referral}

Laparoscopic cholecystectomy was performed in 20 (47.62\%) ( 2 of them were converted to open), and the remaining 22 by open procedure. As per the operating surgeon, the Calot's anatomy could be clearly defined in 19 patients (45.24\%) while 17 (40.48\%) experienced hemorrhage during surgery which obscured the view and operative details could not be traced in the remaining six. At the end of the procedure, bile was seen in the operative field in 12 patients $(33.33 \%)$. Out of these $12(29 \%)$, four were referred immediately, while eight had a delayed referral. Cut section of the gallbladder on was reported to have severe chronic cholecystitis in 20 , acutely inflamed in 8 patients, suspicious of malignancy in 1, gangrenous in 1 and not reported in 12 patients. Intraoperative drains were placed in 31 (73.81\%).

Four patients had suspicion of the injury during cholecystectomy and were referred within 24 hours. The mean and median time of injury detection and referral was $4.32 \pm 2.33$ (range: 0-17) and 3 days respectively.

Thirty-one patients were referred with abdominal drains, which showed bilious fluid in 26, bile and blood in 2, enteric content in 2 and blood in one. Out of 11 patients without drains, eight had multiple bile collections on abdominal imaging (Fig. 2A) while three had bile discharge from the surgical wound of open cholecystectomy. Four (9.6\%) of the patients had diffuse peritonitis and one had melena in addition.

\section{Initial management}

Controlled fistula was observed in 22 of the 31 patients with drain placed at index surgery, while 8 required image
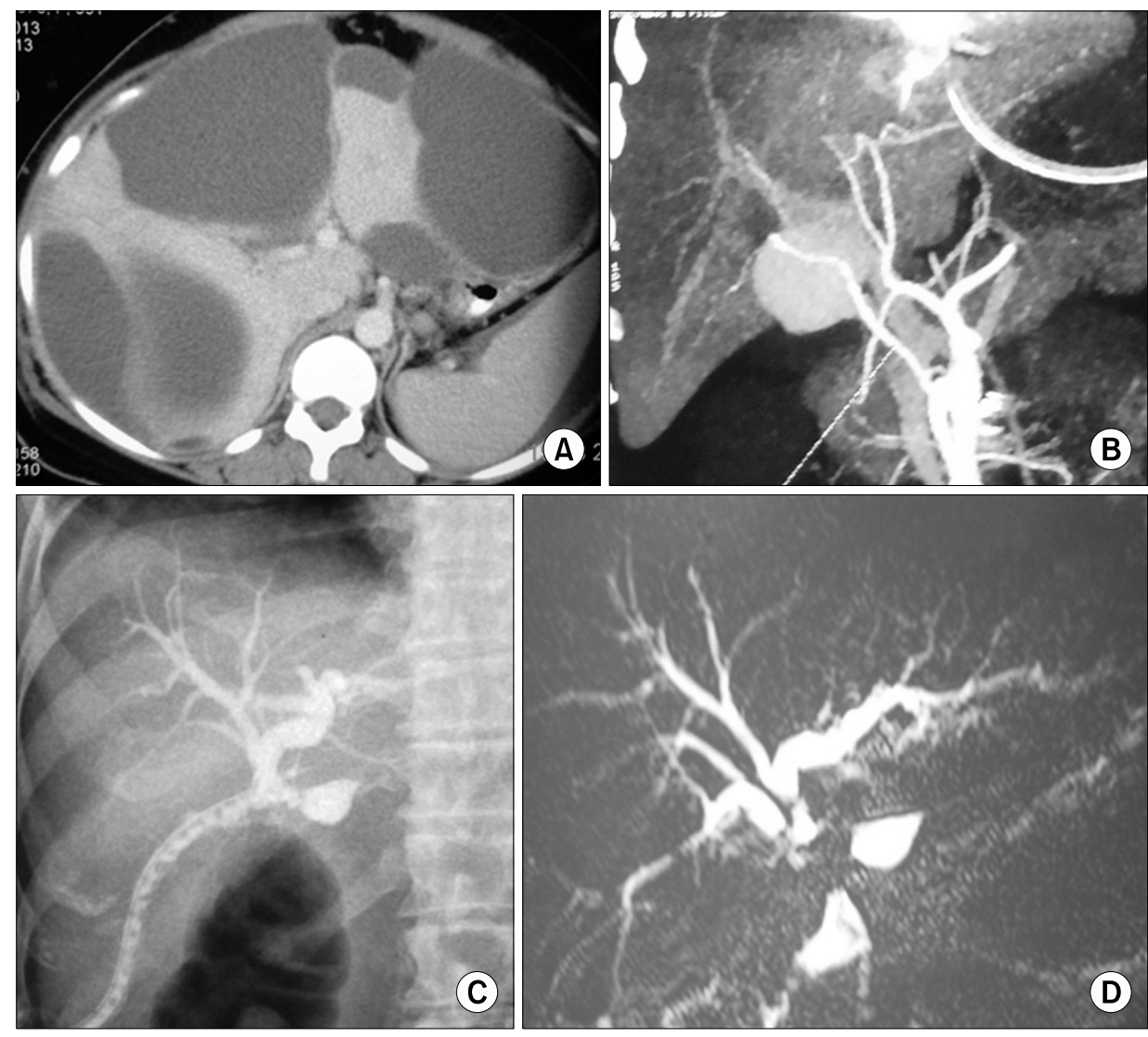

Fig. 2. (A) CT showing multiple bile collections after cholecystectomy. (B) Reconstructed CT image to show pseudoaneurysm arising form replaced right hepatic artery. (C) Contrast study done through drainage tube in patient with persistent fistula shows delineation of the biliary system. Note right posterior sectoral duct is not visualized on this image, which was later seen on MRI (D). This patient had type E5 injury. 
guided percutaneous drains (PCD) and one needed laparotomy. Of the 11 patients referred without drains, six needed image guided PCD and 5 required re-operation.

The drainage was bilious in $36(86 \%)$, bile with blood in $1(2.4 \%)$, only blood in $2(4.8 \%)$, bile in drain with malena in $1(2.4 \%)$ and enteric in $2(4.8 \%)$ patients.

Nine $(21.42 \%) 1$ patients had organ failure at presentation. Six of them had more than one organ failure at admission. Cardiovascular system failure was seen in eight, respiratory failure in six and renal failure in four.

Six patients $(12 \%)$ were re-operated. Five underwent re-laparotomy - two for enteric injury and two for hemorrhage and one for multiple intra-abdominal bile collections; and one was successfully managed with re-laparoscopy.

\section{Management of enteric injuries}

There were enteric injuries in two patients - one duodenal and another had colonic injury during cholecystectomy (injured while taking down the duodenum or colon using cautery current). Patient with duodenal injury had a 2-cmsized perforation in the superior wall of the junction of the $1^{\text {st }}$ and the $2^{\text {nd }}$ parts. He was successfully managed with primary closure, decompressive duodenostomy and feeding jejunostomy. The one with colonic injury had 2.5-cm-sized perforation at the hepatic flexure with fecal peritonitis. He underwent right colectomy and terminal ileostomy, however, he died of multi-organ failure.

\section{Management of hemorrhage}

Two patients presented with hemorrage and bile leak. One had melena and required 4 units of PRBC (packed red blood cell) transfusion. On CT angiogram, pseudoaneurysm of the replaced right hepatic (Fig. 2B) artery was detected. This was not amenable to endotherapy and was managed with surgical bipolar ligation. This patient had concomitant cystic duct blow out which was successfully suture ligated at laparotomy. Another patient presenting with bile and blood from drain was managed with angio-embolization of the right hepatic artery and exteriorization of the biliary fistula.

Two patients had hemorrhage without any evidence of bile collection. One had trocar site bleeding and was operated upon. There was active bleeding from small bowel mesenteric tear (injury occurred while placement of the lumber placement) without any evidence of bowel ischemia. The patient was successfully managed with suture ligationa and closure of mesenteric defect. Another patient was found to have pseudoaneurysm arising from replaced right hepatic artery which was successfully managed with angio-embolization.

\section{Endoscopic retrograde cholangiography (ERC)}

ERC was performed in 17 patients, 10 prior to referral and 7 after admission. Complete cut off of the lower bile duct was observed in 8 patients. Stent was placed in 7 , while in 2 patients there was failure of cannulation. One patient developed post ERC mild pancreatitis. One had concomitant common bile duct stone on ERC which was successfully cleared. Fistula closed shortly after stent placement in 5 patients, while in 2 patients the fistula persisted for 26 and 30 days. Both the patients were found to have right sectoral duct injury.

\section{Mortality}

Six (14.28\%) patients died. Two of the operated patients died - one as a result of multi-organ failure following colonic injury and another due to postoperative myocardial infarction following lavage done for bile peritonitis. Four patients of biliary fistula who had two or more organ failure at admission died as a result multi-organ failure. On univariate and multivariate analysis, age more than 50 years $(8.3 \%$ vs $50 \%, p=0.038$; $\mathrm{RR}=6.00$; $\mathrm{CI}=1.56-23.07)$ and presence of organ failure at admission $(11.1 \%$ vs $83.3 \%, p=0.000 ; \mathrm{RR}=7.5, \mathrm{CI}=2.78-20.20)$ were the determinants of mortality (Table 1).

\section{Fistula closure}

At three months follow up fistula closed in 31 (86.11\%) of 36 patients surviving the acute injury. The mean time for fistula closure was $41.22 \pm 12.3$ days (range 15-90). Fistula closure occurred early in patients with partial injury than with complete injury $(28.89 \pm 2.34$ versus $55.12 \pm$ 4.89 day; $p=0.002)$. All five patients $(13.89 \%)$ with persistent fistula completed biliary transaction.

\section{Sequelae of acute injury}

Of the 36 patients discharged, two patients refused for further follow up. Six (35\%) of the 17 patients were found to have concomitant vascular injury on evaluation. Mag- 
Table 1. Predictors of mortality after cholecystectomy

\begin{tabular}{|c|c|c|c|c|c|}
\hline Parameter & Survived $(\mathrm{N}=36)$ & Died $(\mathrm{N}=6)$ & $\begin{array}{c}\text { Univariate } \\
p \text {-value }\end{array}$ & $\begin{array}{c}\text { Multivariate } \\
p \text {-value }\end{array}$ & Odds ratio $(\mathrm{CI})$ \\
\hline Mean age & $38.77 \pm 11.90$ & $60.50 \pm 7.46$ & 0.80 & & \\
\hline Age $>50$ years & 3 & 3 & 0.007 & 0.038 & $6(1.56-20.07)$ \\
\hline Male:Female & $6: 30$ & $2: 4$ & 0.69 & & \\
\hline Initial surgery laparoscopic:open & $17: 19$ & $3: 3$ & 0.75 & & \\
\hline Timing of referral & $3.80 \pm 0.60$ & $4.33 \pm 1.99$ & 0.18 & & \\
\hline Referral beyond $24 \mathrm{hrs}$ & 22 & 3 & 0.67 & & \\
\hline Nature of injury & & & 0.77 & & \\
\hline Isolated biliary & 31 & 5 & & & \\
\hline Isolated vascular & 2 & 0 & & & \\
\hline Biliary \& vascular & 2 & 0 & & & \\
\hline Enteric & 1 & 1 & & & \\
\hline Need for re-surgery & 4 & 2 & 0.42 & & \\
\hline Organ failure at admission & 4 & 5 & 0.000 & 0.000 & $7.5(2.78-20.2)$ \\
\hline
\end{tabular}

Table 2. Showing the classification of Injuries according to Strasberg ${ }^{10}$

\begin{tabular}{llc}
\hline \multicolumn{1}{c}{ Type } & N & $\begin{array}{c}\text { Persistent } \\
\text { fistula }\end{array}$ \\
\hline A: Cystic or aberrant ducts & $6(19)$ & - \\
C: Aberrant duct without continuity with & $2(6)$ & - \\
the common bile duct* & $2(6)$ & - \\
D: Lateral damage extrahepatic duct & $7(22)$ & 1 \\
E2: Common bile duct $<2 \mathrm{~cm}$ from hep- & $7(1)$ & \\
atic confluence & $11(34)$ & 2 \\
E3: Hepatic confluence & $3(9)$ & 1 \\
E4: Division of right or left hepatic duct & $1(3)$ & 1 \\
E5: stricture with sectoral duct injury & $1(3)$
\end{tabular}

*Both patients had delayed fistula closure

Values in parenthesis indicate percentages

netic Resonance Cholangiopancreaticogram (MRCP) could be done in 34 patients. Patients with persistent fistula underwent evaluation by fistulogram in addition to MRCP (Fig. 2C, D). Two patients with duodenal injury and mesenteric injury had normal cholangiogram. BDI as classified in 32 patients (Table 2) - Partial injury in 10 (31.25\%) and complete stricture in $22(68.75 \%)$. All the patients underwent successful bilio-enteric anastomosis (Roux-en-Y hepatico-jejunostomy) - 17 after the closure of fistula and 5 in the presence of active fistula. None of the patients with active fistula developed bile leak following definitive surgical repair. All patients made an uneventful recovery.

The median follow-up was 57 (40-77) months. Patient with duodenal injury developed incisional hernia which required surgical repair. Two patients died of unrelated cause in the follow up period. All the ten patients with type A, C and D injuries were asymptomatic and had normal LFT. Amongst the 17 patients who underwent repair without fistula - 14 were asymptomatic with normal LFT, two had deranged LFT without any clinical or radiological obstruction, one developed stricture at the hepatico-jejunostomy site and was managed with percutaneous balloon dilatation. Amongst the five patients who underwent repair in the presence of active fistula - three were asymptomatic with normal LFT while the other two had deranged LFT without any clinical or radiological obstruction.

\section{DISCUSSION}

Ever since the introduction of laparoscopic cholecystectomy, there are many reports regarding increasing incidence of bile duct injuries associated with this procedure. $^{2-4}$ Many reports have described the mechanisms and safety measures to be observed during laparoscopic cholecystectomy to prevent this catastrophe. ${ }^{11-14}$ Very few reports have discussed the occurrence of bile duct injuries occurring after open cholecystectomy, probably, due to the fewer number of open procedures being performed. ${ }^{4,9,15,16}$ Open cholecystectomy is usually reserved as a salvage procedure. Contrary to this, in the present audit, we observed that bile duct injury after laparoscopic procedure being outnumbered by the open procedure. Although, the exact number of open or laparoscopic cholecystectomies was not known as ours is a tertiary referral hospital. There were only a small fraction of the patients who were converted to an open procedure. 
More than half of the operative surgeons had a perception of clear dissection in the calot's triangle in both open and laparoscopic surgery. This means there was mis-identification of the bile duct as cystic duct, which has been postulated as the mechanism of injury both in open and laparoscopic surgeries. ${ }^{12,13}$

The exact incidence of enteric injury during cholecystectomy is unknown as it is being reported rarely. In a recent review reporting of complications after laparoscopic cholecystectomy, the entire emphasis was laid on bile duct injuries. ${ }^{17}$ There is not even a single large series which has reported any enteric injury. ${ }^{17}$ Enteric injuries have been reported as anecdotes. ${ }^{18}$ We observed enteric injury in $2(4.76 \%)$ patients. Duodenum and colon being in close proximity to gall bladder are prone to injury either during adhesiolysis or due to inadvertent diathermy trauma. ${ }^{18}$ Detection of enteric injuries can be delayed as these are not suspected at index operation. Diathermy injuries usually have a delayed manifestation. Others have also reported delayed presentation of enteric injuries following cholecystectomy. ${ }^{18}$

The majority of fistula can be lateralized by image guided drainage, only a few will require operative drainage. In the present study, $12 \%$ patients needed operative management to control fistula. Others have reported the need for surgery in acute phase in $<5 \%$ cases. ${ }^{5,15,16}$ Higher rate for surgical intervention was due to the delayed referral and the occurrence of enteric injuries as well. Delayed referral to a specialized center has been associated with increases the morbidity. ${ }^{17}$ We did not observe higher mortality due to delayed referral.

A recent meta-analysis has condemned its routine usage of intra-abdominal drainage tube. ${ }^{19}$ Most of the studies have failed to demonstrate the benefit of placing drainage tube routinely. ${ }^{19,20}$ This is probably due to a very low incidence of complications. To demonstrate $20 \%$ benefit of drain, one needs at least 96 patients with bile duct injuries randomized to each group. If we assume $0.5 \%$ incidence of bile duct injuries, we can imagine a huge number of cholecystectomies required to produce so many injuries. However, the use of selective drainage tube placement is a good policy. In the present study, prior drain tube placement avoided the need for percutnaeous drainage in $71 \%$ (22 out of 31 ) patients.

There are many reports describing the association of vascular injury with biliary injury. ${ }^{4-6,9,11,14}$ Late sequelae of associated vascular injury and its impact on long term biliary repair have been well described in literature. ${ }^{4,5,11,14}$ However, except for a few reports of pseudoaneurysms of hepatic and cystic arteries, not much has been described in the setting of acute injury. ${ }^{21,22}$ In the present study, $10 \%$ of patients presented with acute hemorrhage after cholecystectomy. Besides, vascular causes, we also observed hemorrhage from bowel mesentery arising as a result of trocar injury. Others have implicated antiplatelet drugs as an etiological factor for the occurrence of hemorrhage. ${ }^{23}$

We observed 14\% mortality in acute bile duct injury. This mortality should directly be attributed to the consequence of cholecystectomy. However, others have reported mortality associated with cholecystectomy to be related to associated co-morbid conditions and fraility of the individual. Laparoscopic cholecystectomy in large series in elderly have been shown to be safe. ${ }^{24,25}$ However, caution should be observed in every cholecystectomy as a potential to cause bile leak and its consequence.

Persistent fistula at the end of three months was seen in $14 \%$ patients. Others have reported a lower fistula rate. ${ }^{26}$ Such presence of fistula carries its own morbidity in terms of fluid and bile loss. In addition, repairs in the presence of fistula are usually fraught with higher complications. A fistula which persists at 3 months is unlikely to close subsequently and waiting beyond this time will be futile. So definitive repairs should be undertaken at 3 months to limit the morbidity associated persistent fistula. We observed a comparable long-term outcome of the patients who were operated in the presence of active fistula. We have proposed the algorithm in the management of post cholecystectomy acute injury which encompass associated organ injury and vascular injury in addition to bile duct injury.

In conclusion, post cholecystectomy acute injury does not limit itself to bile duct or vascular injury but it can traumatize adjacent hollow viscus or mesentery. It is important to diagnose and intervene early in enteric injury. The presentation and management should be followed as per the proposed algorithm. Age more than 50 years and presence of organ failure were the predictors of mortality. Therefore, acute injury should be managed with a multi-disciplinary approach. 


\section{REFERENCES}

1. McKinley SK, Brunt LM, Schwaitzberg SD. Prevention of bile duct injury: the case for incorporating educational theories of expertise. Surg Endosc 2014;28:3385-3391.

2. Suo T, Chen L, Liu H, Ni X, Shen S, Wang Y, et al. Management for a complicated biliary stricture after iatrogenic bile duct injury. J Vis Surg 2017;3:33.

3. Kohn JF, Trenk A, Kuchta K, Lapin B, Denham W, Linn JG, et al. Characterization of common bile duct injury after laparoscopic cholecystectomy in a high-volume hospital system. Surg Endosc 2018;32:1184-1191.

4. Karvonen J, Salminen P, Grönroos JM. Bile duct injuries during open and laparoscopic cholecystectomy in the laparoscopic era: alarming trends. Surg Endosc 2011;25:2906-2910.

5. Otto W, Sierdziński J, Smaga J, Dudek K, Zieniewicz K. Longterm effects and quality of life following definitive bile duct reconstruction. Medicine (Baltimore) 2018;97:e12684.

6. Stilling NM, Fristrup C, Wettergren A, Ugianskis A, Nygaard $\mathrm{J}$, Holte $\mathrm{K}$, et al. Long-term outcome after early repair of iatrogenic bile duct injury. A national Danish multicentre study. HPB (Oxford) 2015;17:394-400.

7. Andersson R, Eriksson K, Blind PJ, Tingstedt B. Iatrogenic bile duct injury--a cost analysis. HPB (Oxford) 2008;10:416-419.

8. Scurr JR, Brigstocke JR, Shields DA, Scurr JH. Medicolegal claims following laparoscopic cholecystectomy in the UK and Ireland. Ann R Coll Surg Engl 2010;92:286-291.

9. Mishra PK, Saluja SS, Nayeem M, Sharma BC, Patil N. Bile duct injury-from injury to repair: an analysis of management and outcome. Indian J Surg 2015;77(Suppl 2):536-542.

10. Strasberg SM, Hertl M, Soper NJ. An analysis of the problem of biliary injury during laparoscopic cholecystectomy. J Am Coll Surg 1995;180:101-125.

11. Balla A, Quaresima S, Corona M, Lucatelli P, Fiocca F, Rossi $\mathrm{M}$, et al. ATOM classification of bile duct injuries during laparoscopic cholecystectomy: analysis of a single institution experience. J Laparoendosc Adv Surg Tech A 2019;29:206-212.

12. Iwashita Y, Hibi T, Ohyama T, Umezawa A, Takada T, Strasberg $\mathrm{SM}$, et al. Delphi consensus on bile duct injuries during laparoscopic cholecystectomy: an evolutionary cul-de-sac or the birth pangs of a new technical framework? J Hepatobiliary Pancreat Sci 2017;24:591-602.

13. Dissanaike S. A step-by-step guide to laparoscopic subtotal fenestrating cholecystectomy: a damage control approach to the difficult gallbladder. J Am Coll Surg 2016;223:e15-e18.

14. Fingerhut A, Dziri C, Garden OJ, Gouma D, Millat B, Neugebauer E, et al. ATOM, the all-inclusive, nominal EAES classification of bile duct injuries during cholecystectomy. Surg Endosc 2013; 27:4608-4619.

15. Gorsi U, Gupta P, Kalra N, Kang M, Singh R, Gupta R, et al. Multidetector computed tomography evaluation of post cholecystectomy complications: a tertiary care center experience. Trop Gastroenterol 2015;36:236-243.

16. Martinez-Lopez S, Upasani V, Pandanaboyana S, Attia M, Toogood $\mathrm{G}$, Lodge $\mathrm{P}$, et al. Delayed referral to specialist centre increases morbidity in patients with bile duct injury (BDI) after laparoscopic cholecystectomy (LC). Int J Surg 2017;44:82-86.

17. Alexander HC, Bartlett AS, Wells CI, Hannam JA, Moore MR, Poole GH, et al. Reporting of complications after laparoscopic cholecystectomy: a systematic review. HPB (Oxford) 2018;20: 786-794.

18. Machado NO. Duodenal injury post laparoscopic cholecystectomy: Incidence, mechanism, management and outcome. World J Gastrointest Surg 2016;8:335-344.

19. Wong CS, Cousins G, Duddy JC, Walsh SR. Intra-abdominal drainage for laparoscopic cholecystectomy: a systematic review and meta-analysis. Int J Surg 2015;23:87-96.

20. Qiu J, Li M. Nondrainage after laparoscopic cholecystectomy for acute calculous cholecystitis does not increase the postoperative morbidity. Biomed Res Int 2018;2018:8436749.

21. Machado NO, Al-Zadjali A, Kakaria AK, Younus S, Rahim MA, Al-Sukaiti R. Hepatic or cystic artery pseudoaneurysms following a laparoscopic cholecystectomy: literature review of aetiopathogenesis, presentation, diagnosis and management. Sultan Qaboos Univ Med J 2017;17:e135-e146.

22. Petrou A, Brennan N, Soonawalla Z, Silva MA. Hemobilia due to cystic artery stump pseudoaneurysm following laparoscopic cholecystectomy: case presentation and literature review. Int Surg 2012;97:140-144.

23. Strömberg J, Sandblom G. Impact of comorbidity and prescription drugs on haemorrhage in cholecystectomy. World J Surg 2017;41:1985-1992.

24. Mastalerz K, Kenig J, Olszewska U, Michalik C. The Surgical Apgar Score and frailty as outcome predictors in short- and long-term evaluation of fit and frail older patients undergoing elective laparoscopic cholecystectomy - a prospective cohort study. Wideochir Inne Tech Maloinwazyjne 2018;13:350-357.

25. Ekici U, Yılmaz S, Tatlı F. Comparative analysis of laparoscopic cholecystectomy performed in the elderly and younger patients: should we abstain from laparoscopic cholecystectomy in the elderly? Cureus 2018;10:e2888.

26. Carr-Locke AD. 'Biliary stenting alone versus biliary stenting plus sphincterotomy for the treatment of post-laparoscopic cholecystectomy bile leaks'. Eur J Gastroenterol Hepatol 2006;18: 1053-1055. 